\title{
Imprecision and Preferences in Interpretation of Verbal Probabilities in Health: a Systematic Review
}

\author{
Katerina Andreadis, $\mathrm{MS}^{7}$ (D), Ethan Chan, PharmD, $\mathrm{MS}^{1,2}$, Minha Park, $\mathrm{MS}^{\mathrm{l}, 2}$, \\ Natalie C Benda, $\mathrm{PhD}^{7}$, Mohit M Sharma, $\mathrm{MPH}^{7}$, Michelle Demetres, $\mathrm{MS}^{3}$, \\ Diana Delgado, $M L S^{3}$, Elizabeth Sigworth, $B A^{4}$, Qingxia Chen, $P h D^{4}$, Andrew Liu, $B S^{5}$, \\ Lisa Grossman Liu, MPhil , Marianne Sharko, MD, MS ${ }^{7}$, Brian J Zikmund-Fisher, PhD ${ }^{5}$, and \\ Jessica S Ancker, MPH, PhD ${ }^{1,7}$
}

\begin{abstract}
'Department of Population Health Sciences, Weill Cornell Medicine, New York, NY, USA; ${ }^{2}$ Memorial Sloan Kettering Cancer Center, New York, NY, USA; ${ }^{3}$ Samuel J Wood Library, Weill Cornell Medicine, New York, NY, USA; ${ }^{4}$ Department of Biostatistics, Vanderbilt University Medical Center, Nashville, TN, USA; ${ }^{5}$ Department of Health Behavior and Health Education, University of Michigan School of Public Health, Ann Arbor, MI, USA; ${ }^{6}$ Department of Biomedical Informatics, Columbia University Vagelos School of Medicine, New York, NY, USA; ${ }^{7}$ Department of Biomedical Informatics, Vanderbilt University Medical Center, Nashville, TN, USA.
\end{abstract}

INTRODUCTION: Many health providers and communicators who are concerned that patients will not understand numbers instead use verbal probabilities (e.g., terms such as "rare" or "common") to convey the gist of a health message.

OBJECTIVE: To assess patient interpretation of and preferences for verbal probability information in health contexts.

METHODS: We conducted a systematic review of literature published through September 2020. Original studies conducted in English with samples representative of lay populations were included if they assessed health-related information and elicited either (a) numerical estimates of verbal probability terms or (b) preferences for verbal vs. quantitative risk information.

RESULTS: We identified 33 original studies that referenced 145 verbal probability terms, 45 of which were included in at least two studies and 19 in three or more. Numerical interpretations of each verbal term were extremely variable. For example, average interpretations of the term "rare" ranged from 7 to $21 \%$, and for "common," the range was 34 to $71 \%$. In a subset of 9 studies, lay estimates of verbal probability terms were far higher than the standard interpretations established by the European Commission for drug labels. In 10 of 12 samples where preferences were elicited, most participants preferred numerical information, alone or in combination with verbal labels.

CONCLUSION: Numerical interpretation of verbal probabilities is extremely variable and does not correspond well to the numerical probabilities established by expert panels. Most patients appear to prefer quantitative risk information, alone or in combination with verbal labels. Health professionals should be aware that avoiding numeric information to describe risks may not match patient preferences, and that patients interpret verbal risk terms in a highly variable way.

Received February 24, 2021

Accepted July 14, 2021

Published online August 6, 2021
KEY WORDS: risk communication; patient-provider communication; health literacy; health numeracy.

J Gen Intern Med 36(12):3820-9 DOI: $10.1007 / \mathrm{s} 11606-021-07050-7$ (C) The Author(s) 2021

\section{INTRODUCTION}

Although probabilities of risk and benefit are often important components of medical information, it is well established that many patients have low numeracy, which may impair their ability to understand or make decisions on the basis of numerical information. ${ }^{1-5}$ As a result, healthcare providers may believe that patients will not be able to use numerical information or that they prefer words to numbers. ${ }^{6}$ In one survey, only about $35 \%$ of ob-gyns reported routinely using numbers when talking with patients about screening tests, with the remainder preferring verbal terms such as "low risk" or labels such as "normal/abnormal.", A different survey found that family physicians used numbers or quantitative graphics to describe cardiovascular risk in only $27 \%$ of patient visits; in the remainder of the visits, the physician used verbal risk terms only. ${ }^{8}$ Healthcare providers' use of quantitative risk information (in the form of numbers or graphics) appears to be related to factors including their own numeracy, their perception of the patients' numeracy, and the gender of both the provider and the patient. ${ }^{8,9}$

In non-medical domains, risk communication research has demonstrated that a major limitation of relying on verbal probability terms is that they are interpreted in highly variable ways by the recipients of the information. ${ }^{10-13}$ An additional source of uncertainty in verbal risk communication is that the speaker may choose different verbal probability terms according to their opinion and previous experiences, and this choice is likely to in turn influence the recipient's judgment. ${ }^{14}$ One study found that choice of verbal terms is even influenced by 
politeness, so that polite speakers generally communicated lower risk magnitudes than less polite ones. ${ }^{15}$

However, extrapolating findings from non-medical contexts to medical ones may be problematic, given the domainspecific nature of risk perceptions and behaviors. ${ }^{10,16} \mathrm{We}$ therefore consider it important to assess the impact of verbal probability expressions in medical and health contexts only. Also, in light of healthcare professionals' persistent use of verbal-only risk communications, we believe it is important to clarify whether patients in fact prefer verbal descriptions of risk to numerical ones.

Therefore, the objective of this study was to review the existing literature to synthesize evidence on patient interpretation of and preference for verbal probabilities in health and medical communication.

\section{METHODS}

This study analyzed a subset of articles from a large systematic review of experimental and quasi-experimental research contrasting different formats (numerical, graphical, and verbal) for presenting health-related quantitative information to the lay public. The review included both probabilities (such as health risks) and quantities (such as laboratory values and environmental data) and was limited to studies measuring quantitative outcomes including preference, comprehension, and decisions.

We performed the systematic review following the Preferred Reporting Items for Systematic Reviews and Meta-Analyses (PRISMA) statement (Fig. 1). ${ }^{17}$ In adherence to these guidelines, we registered a protocol in PROSPERO (registration \#CRD42018086270). Two experienced librarians constructed a systematic approach to search Ovid MEDLINE, Ovid Embase, the Cochrane Library (Wiley), CINAHL (EBSCO), ERIC (ProQuest), PsycINFO (EBSCO), and the ACM Digital Library, from inception to January 2019, with an update on September 10, 2020. See Appendix 1 for the search strategy for Ovid MEDLINE. To supplement these results, we identified the top 4 most common journals from database searches (Medical Decision Making, Patient Education and Counseling, Risk Analysis, and Journal of Health Communication) and hand-searched their tables of contents in their entirety from 2008 up to 2019. For articles selected for inclusion in this study, we pulled and screened reference lists and citing articles from Scopus (Elsevier). Searches produced a total of 37,839 articles. After de-duplication, two independent reviewers screened 26,793 titles and abstracts using Covidence systematic review web software (Covidence.org, Melbourne, Australia). We then assessed 1500 articles for full-text review, with discrepancies resolved by consensus or third reviewer.

Pairs of reviewers performed full-text review. A threemember verbal probabilities team (KA, EC, MP) reviewed all articles studying verbal probability terms such as "rare," "common," or "likely" (76 articles) and included publications meeting the following criteria:

1. Original studies presenting participants with healthrelated information.

2. Sample was adult laypeople without expertise in a health profession (the study was included if at least one subsample met these inclusion criteria and if the results for the subsample were reported separately).

3. Quantitative assessments of outcomes included:
a. Numerical estimates of the meaning of the verbal term, and/or
b. Preferences for verbal vs numeric terms.

4. Verbal probabilities expressed in English. (The larger review did not include a language restriction. However, for this analysis of verbal probabilities, we limited research to studies conducted in English to ensure that interpretations of the verbal terms would not be confounded by potential differences in translation.)

Three reviewers extracted data from the included articles using a custom-developed Qualtrics instrument. Data extracted included the main question or comparison, the outcomes measured (either numerical interpretation of the probability terms, preferences, or both), the sample size, and the population recruited. We captured details about the stimuli including the specific verbal terms studied; whether the probability was chance of disease, medication side effects, or adverse effects of a procedure; the general health condition or domain if specified; and the severity of the health event if specified, e.g., mild or severe.

In recording the outcome of numerical estimates of a verbal probability, we recorded sample sizes, mean estimates, ranges, and (where provided) either standard deviations or 95\% confidence intervals. In the list of verbal terms, we did not distinguish between adjectival and adverbial forms (e.g., rare and rarely). A subset of studies examined the terms listed in the European Commission (EC) guidelines, which standardize the use of verbal probability terms to be used for medication side effects. ${ }^{18}$ (For example, these guidelines specify that the term "rare" is to be used for events with probabilities between 0.01 and $0.1 \%$, and the term "very rare" for probabilities lower than $<0.01 \% .^{18}$ ) For these studies, we also recorded a "correct/ incorrect" flag by whether respondents provided a numeric probability within the range specified by the EC for that term. In recording the outcome of preference, we recorded the proportions who reported preferring words, numbers, both, other, or no preference.

To pool the estimated probabilities from individual studies, we included only studies that reported either confidence intervals or standard deviations. We performed meta-analysis of single means, choosing a random effects model to account for heterogeneity that is due to both random error and potential systematic differences between studies. ${ }^{19}$ The inverse variance method was used for pooling study estimates, ${ }^{20}$ and the 


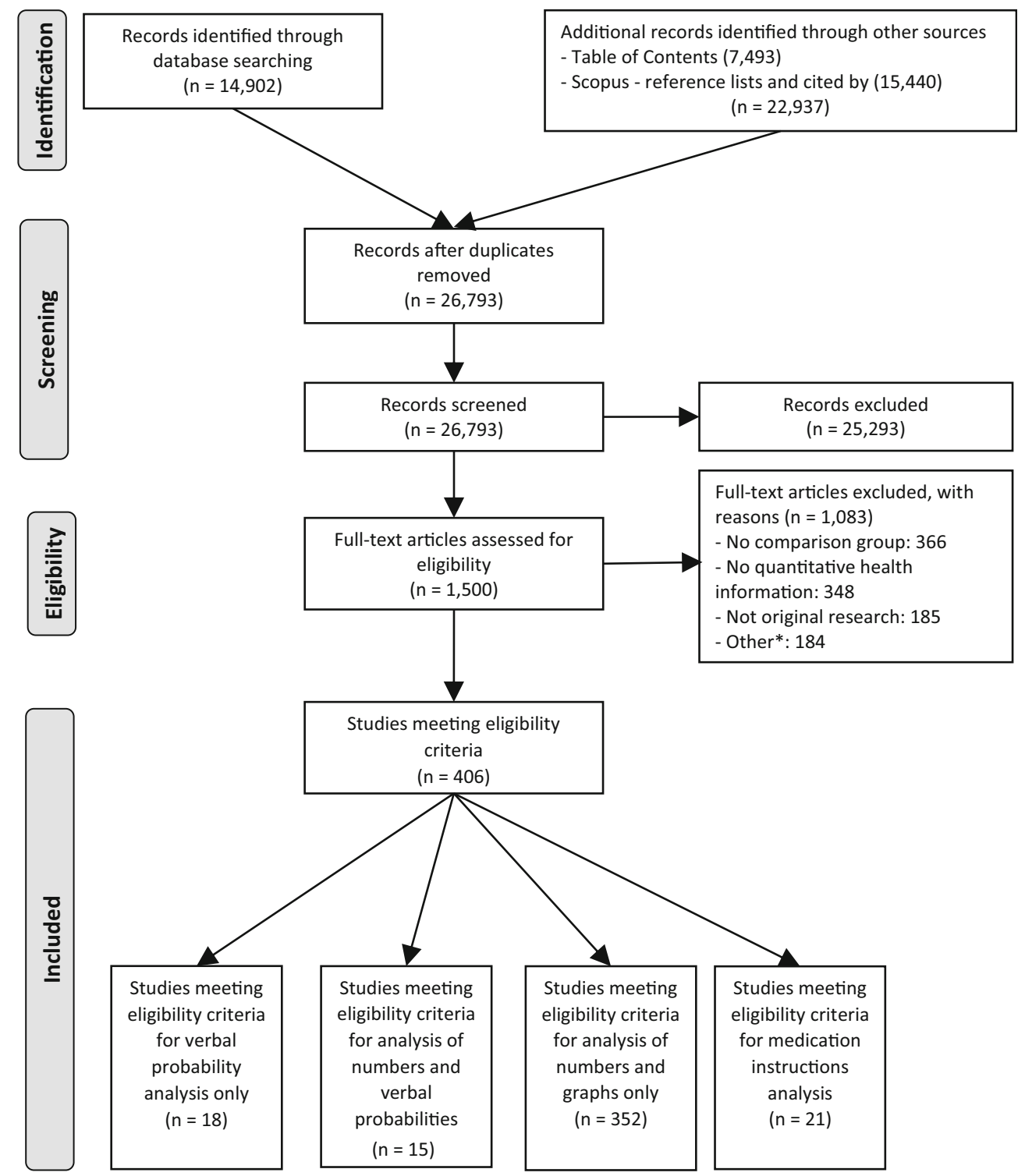

Figure 1 PRISMA flow diagram. *Other: duplicate dataset, no quantitative evaluation metric, insufficient detail to extract, not adults, experiments designed to understand beliefs not response to information, no full text available, test of education method, scale development/ calibration, decision was not a personal health/medical decision, non-patient (health professional), comparator was different terms for cancer not different formats, verbal probabilities not in English.

DerSimonian-Laird estimator of tau-squared (a measure of variance representing between-study heterogeneity) was used to adjust standard errors. ${ }^{21}$ For confidence intervals around tau-squared and tau, the Jackson method was used. ${ }^{22}$ Forest plots were generated of the individual study means and confidence intervals alongside the pooled random effects estimates. The meta-analysis and figures were generated using $\mathrm{R}$ version 4.0.5 and the "meta" package version 4.18-1. ${ }^{23,24}$

Risk of bias assessment is meant to capture the quality of each study and the likelihood of producing biased results. To assess risk of bias in this review, we adapted criteria from the AHRQ Methods Guide for Comparative Effectiveness Reviews and Cochrane Handbook for Systematic Reviews of Interventions. ${ }^{25,26}$ Pairs of team members scored each study on sample selection, randomization process, protocol deviations, measurement of covariates, missing data, and presence of other potential biases. Scoring conflicts were resolved in consensus meetings. The score was then classified as low, moderate, or high risk of bias.

\section{RESULTS}

As shown in Figure 1, the systematic literature search resulted in 406 studies in 4 subsets, focusing on verbal probabilities only, verbal and numerical probabilities, numbers and graphs only, and medication instructions. The current paper includes the first and second subsets: verbal probabilities only, and verbal versus numerical probabilities.

The final sample included 33 studies, which were published between 1967 and 2020 (Table 1). Of the studies, 14 were conducted in the UK, 12 in the USA, 5 in 
Table 1 Articles on Verbal Probabilities and Characteristics Collected

\begin{tabular}{|c|c|c|c|c|c|c|c|c|}
\hline $\begin{array}{l}\text { Authors and } \\
\text { year }\end{array}$ & Research question & $\begin{array}{l}\text { Primary } \\
\text { outcome } \\
\text { measured }\end{array}$ & $\begin{array}{l}\text { Sample } \\
\text { size* }\end{array}$ & $\begin{array}{l}\text { Sample } \\
\text { description* }\end{array}$ & $\begin{array}{l}\text { Health } \\
\text { condition or } \\
\text { situation }\end{array}$ & $\begin{array}{l}\text { Severity of } \\
\text { health event } \\
\text { specified? }\end{array}$ & $\begin{array}{l}\text { Study } \\
\text { of EC } \dot{\dagger} \\
\text { terms? }\end{array}$ & $\begin{array}{l}\text { Risk of } \\
\text { bias } \\
\text { assessment }\end{array}$ \\
\hline $\begin{array}{l}\text { Lichtenstein } \\
\text { and Newman } \\
196727\end{array}$ & $\begin{array}{l}\text { To assess } \\
\text { numerical estimates } \\
\text { and symmetry of } \\
\text { interpretation } \\
\text { of "mirror image" } \\
\text { pairs of terms (e.g., } \\
\text { "quite likely"--" } \\
\text { "quite unlikely") }\end{array}$ & $\begin{array}{l}\text { Numerical } \\
\text { estimates }\end{array}$ & 188 & Adult males & Not specified & No & No & $\begin{array}{l}\text { A little } \\
\text { concern }\end{array}$ \\
\hline $\begin{array}{l}\text { Budescu et al. } \\
1985^{28}\end{array}$ & $\begin{array}{l}\text { To assess } \\
\text { variability in the } \\
\text { mapping of phrases } \\
\text { to numbers }\end{array}$ & $\begin{array}{l}\text { Numerical } \\
\text { estimates }\end{array}$ & 32 & $\begin{array}{l}\text { Faculty and } \\
\text { graduate students } \\
\text { of a university }\end{array}$ & Not specified & No & No & $\begin{array}{l}\text { Moderate } \\
\text { concern }\end{array}$ \\
\hline $\begin{array}{l}\text { Reagan et al. } \\
1989^{29}\end{array}$ & $\begin{array}{l}\text { To map verbal } \\
\text { probability words } \\
\text { to numbers }\end{array}$ & $\begin{array}{l}\text { Numerical } \\
\text { estimates }\end{array}$ & 100 & $\begin{array}{l}\text { Undergraduate } \\
\text { students }\end{array}$ & Not specified & No & No & $\begin{array}{l}\text { Moderate } \\
\text { concern }\end{array}$ \\
\hline $\begin{array}{l}\text { Shaw and } \\
\text { Dear } 1990^{30}\end{array}$ & $\begin{array}{l}\text { To evaluate } \\
\text { understanding of } \\
\text { probability } \\
\text { expressions and } \\
\text { preference for } \\
\text { receiving } \\
\text { information }\end{array}$ & $\begin{array}{l}\text { Numerical } \\
\text { estimates, } \\
\text { format } \\
\text { preference }\end{array}$ & 100 & $\begin{array}{l}\text { Adult female } \\
\text { parents }\end{array}$ & $\begin{array}{l}\text { Aspects of } \\
\text { neonatal care }\end{array}$ & No & No & No concern \\
\hline $\begin{array}{l}\text { Weber and } \\
\text { Hilton } 1990^{31}\end{array}$ & $\begin{array}{l}\text { To examine the } \\
\text { role of context in } \\
\text { the interpretation of } \\
\text { probability words }\end{array}$ & $\begin{array}{l}\text { Numerical } \\
\text { estimates }\end{array}$ & 85 & $\begin{array}{l}\text { Undergraduate } \\
\text { students }\end{array}$ & $\begin{array}{l}\text { Varying } \\
\text { disease types } \\
\text { and side } \\
\text { effects }\end{array}$ & $\begin{array}{l}\text { Some } \\
\text { specified as } \\
\text { severe life- } \\
\text { threatening } \\
\text { events, others } \\
\text { unspecified }\end{array}$ & No & $\begin{array}{l}\text { Moderate } \\
\text { concern }\end{array}$ \\
\hline $\begin{array}{l}\text { Freeman et al. } \\
1992^{6}\end{array}$ & $\begin{array}{l}\text { To identify } \\
\text { patients' preferred } \\
\text { risk language and } \\
\text { physicians' } \\
\text { predictions about } \\
\text { patient preferences }\end{array}$ & $\begin{array}{l}\text { Format } \\
\text { preference }\end{array}$ & 208 & $\begin{array}{l}\text { Adult female } \\
\text { patients with } \\
\text { children from } \\
\text { family practices }\end{array}$ & Vaccine risk & No & No & $\begin{array}{l}\text { Moderate } \\
\text { concern }\end{array}$ \\
\hline $\begin{array}{l}\text { Woloshin } \\
\text { et al. } 1994^{32}\end{array}$ & $\begin{array}{l}\text { To assess patients' } \\
\text { interpretation of } \\
\text { probability terms }\end{array}$ & $\begin{array}{l}\text { Numerical } \\
\text { estimates; } \\
\text { format } \\
\text { preference }\end{array}$ & 307 & $\begin{array}{l}\text { Adult patients } \\
\text { from a family } \\
\text { practice }\end{array}$ & $\begin{array}{l}\text { Medication } \\
\text { side effect or } \\
\text { complication } \\
\text { risk from } \\
\text { procedure }\end{array}$ & $\begin{array}{l}\text { Minor vs } \\
\text { major } \\
\text { complications }\end{array}$ & No & $\begin{array}{l}\text { Moderate } \\
\text { concern }\end{array}$ \\
\hline $\begin{array}{l}\text { Hallowell } \\
\text { et al. } 1997{ }^{33}\end{array}$ & $\begin{array}{l}\text { To evaluate female } \\
\text { patient preferences } \\
\text { in formats used to } \\
\text { present risk } \\
\text { information during } \\
\text { genetic counseling } \\
\text { for breast } \\
\text { and ovarian cancer }\end{array}$ & $\begin{array}{l}\text { Format } \\
\text { preference }\end{array}$ & 43 & $\begin{array}{l}\text { Adult female } \\
\text { patients } \\
\text { presenting for } \\
\text { genetic } \\
\text { counseling in } \\
\text { cancer clinic }\end{array}$ & $\begin{array}{l}\text { Breast and } \\
\text { ovarian cancer } \\
\text { risks }\end{array}$ & No & No & $\begin{array}{l}\text { A little } \\
\text { concern }\end{array}$ \\
\hline $\begin{array}{l}\text { Franic et al. } \\
2000^{34}\end{array}$ & $\begin{array}{l}\text { To evaluate format } \\
\text { preference in } \\
\text { patient medication } \\
\text { package inserts }\end{array}$ & $\begin{array}{l}\text { Format } \\
\text { preference }\end{array}$ & 74 & $\begin{array}{l}\text { Adult female } \\
\text { patients from } \\
\text { academic } \\
\text { university }\end{array}$ & $\begin{array}{l}\text { Adverse drug } \\
\text { reactions }\end{array}$ & No & No & $\begin{array}{l}\text { High } \\
\text { concern }\end{array}$ \\
\hline $\begin{array}{l}\text { Biehl et al. } \\
2001^{35}\end{array}$ & $\begin{array}{l}\text { To compare the } \\
\text { interpretation of } \\
\text { probability terms of } \\
\text { adults } \\
\text { with adolescents }\end{array}$ & $\begin{array}{l}\text { Numerical } \\
\text { estimates }\end{array}$ & 34 & $\begin{array}{l}\text { Adults from a } \\
\text { community } \\
\text { center }\end{array}$ & Not specified & No & No & $\begin{array}{l}\text { A little } \\
\text { concern }\end{array}$ \\
\hline $\begin{array}{l}\text { Kaplowitz } \\
\text { et al. } 2002^{36}\end{array}$ & $\begin{array}{l}\text { To evaluate how } \\
\text { patients want, } \\
\text { request, and } \\
\text { receive cancer } \\
\text { prognosis } \\
\text { information }\end{array}$ & $\begin{array}{l}\text { Format } \\
\text { preference }\end{array}$ & 352 & $\begin{array}{l}\text { Patients from the } \\
\text { American Cancer } \\
\text { Society } \\
\text { (ACS) mailing } \\
\text { list in Michigan, } \\
\text { US }\end{array}$ & $\begin{array}{l}\text { Cancer } \\
\text { prognosis } \\
\text { information }\end{array}$ & No & No & $\begin{array}{l}\text { A little } \\
\text { concern }\end{array}$ \\
\hline $\begin{array}{l}\text { Berry et al. } \\
2002^{37}\end{array}$ & $\begin{array}{l}\text { To assess the } \\
\text { interpretation of } \\
\text { verbal probability } \\
\text { descriptors }\end{array}$ & $\begin{array}{l}\text { Numerical } \\
\text { estimates }\end{array}$ & 268 & $\begin{array}{l}\text { Undergraduate } \\
\text { and graduate } \\
\text { students }\end{array}$ & $\begin{array}{l}\text { Throat } \\
\text { infection or ear } \\
\text { infection; } \\
\text { fictitious } \\
\text { medication } \\
\text { side effect }\end{array}$ & $\begin{array}{l}\text { Mild vs severe } \\
\text { side effects }\end{array}$ & Yes & $\begin{array}{l}\text { Moderate } \\
\text { concern }\end{array}$ \\
\hline $\begin{array}{l}\text { Berry et al. } \\
2003^{38}\end{array}$ & $\begin{array}{l}\text { To compare the } \\
\text { understanding of } \\
\text { verbal and } \\
\text { numerical }\end{array}$ & $\begin{array}{l}\text { Numerical } \\
\text { estimates }\end{array}$ & 360 & $\begin{array}{l}\text { Adults from } \\
\text { various public } \\
\text { settings }\end{array}$ & $\begin{array}{l}\text { Fictitious } \\
\text { medication } \\
\text { side effect }\end{array}$ & $\begin{array}{l}\text { Mild vs severe } \\
\text { side effects }\end{array}$ & Yes & $\begin{array}{l}\text { A little } \\
\text { concern }\end{array}$ \\
\hline
\end{tabular}


Table 1. (continued)

\begin{tabular}{|c|c|c|c|c|c|c|c|c|}
\hline $\begin{array}{l}\text { Authors and } \\
\text { year }\end{array}$ & Research question & $\begin{array}{l}\text { Primary } \\
\text { outcome } \\
\text { measured }\end{array}$ & $\begin{array}{l}\text { Sample } \\
\text { size* }^{*}\end{array}$ & $\begin{array}{l}\text { Sample } \\
\text { description* }\end{array}$ & $\begin{array}{l}\text { Health } \\
\text { condition or } \\
\text { situation }\end{array}$ & $\begin{array}{l}\text { Severity of } \\
\text { health event } \\
\text { specified? }\end{array}$ & $\begin{array}{l}\text { Study } \\
\text { of EC } \dagger \\
\text { terms? }\end{array}$ & $\begin{array}{l}\text { Risk of } \\
\text { bias } \\
\text { assessment }\end{array}$ \\
\hline & $\begin{array}{l}\text { descriptions of } \\
\text { medication side } \\
\text { effects }\end{array}$ & & & & & & & \\
\hline $\begin{array}{l}\text { Budescu et al. } \\
2003^{39}\end{array}$ & $\begin{array}{l}\text { To determine the } \\
\text { directionality of } \\
\text { probability phrases }\end{array}$ & $\begin{array}{l}\text { Numerical } \\
\text { estimates }\end{array}$ & 27 & $\begin{array}{l}\text { Undergraduate } \\
\text { students }\end{array}$ & $\begin{array}{l}\text { Medical } \\
\text { context; } \\
\text { general } \\
\text { medication } \\
\text { administration }\end{array}$ & No & No & $\begin{array}{l}\text { Moderate } \\
\text { concern }\end{array}$ \\
\hline $\begin{array}{l}\text { Davey et al. } \\
2003^{40}\end{array}$ & $\begin{array}{l}\text { To evaluate } \\
\text { women's } \\
\text { understanding of } \\
\text { diagnostic test } \\
\text { results }\end{array}$ & $\begin{array}{l}\text { Numerical } \\
\text { estimates }\end{array}$ & 37 & $\begin{array}{l}\text { Adult women } \\
\text { who had } \\
\text { previously } \\
\text { participated in a } \\
\text { population } \\
\text { survey }\end{array}$ & $\begin{array}{l}\text { Breast cancer } \\
\text { risk }\end{array}$ & No & No & $\begin{array}{l}\text { A little } \\
\text { concern }\end{array}$ \\
\hline $\begin{array}{l}\text { Lobb et al. } \\
2003^{41}\end{array}$ & $\begin{array}{l}\text { To evaluate how } \\
\text { women wanted } \\
\text { their risk of breast } \\
\text { cancer } \\
\text { to be described in } \\
\text { consultation }\end{array}$ & $\begin{array}{l}\text { Format } \\
\text { preference }\end{array}$ & 193 & $\begin{array}{l}\text { Adult women } \\
\text { from cancer } \\
\text { clinics }\end{array}$ & $\begin{array}{l}\text { Breast cancer } \\
\text { risk }\end{array}$ & No & No & $\begin{array}{l}\text { Moderate } \\
\text { concern }\end{array}$ \\
\hline $\begin{array}{l}\text { Berry et al. } \\
2004^{42}\end{array}$ & $\begin{array}{l}\text { To evaluate } \\
\text { people's } \\
\text { interpretation of } \\
\text { EC verbal } \\
\text { descriptors } \\
\text { for medication side } \\
\text { effect risks }\end{array}$ & $\begin{array}{l}\text { Numerical } \\
\text { estimates }\end{array}$ & 188 & $\begin{array}{l}\text { Adults from } \\
\text { various public } \\
\text { places }\end{array}$ & $\begin{array}{l}\text { Over-the- } \\
\text { counter } \\
\text { painkiller } \\
\text { medication } \\
\text { side effects }\end{array}$ & No & Yes & $\begin{array}{l}\text { A little } \\
\text { concern }\end{array}$ \\
\hline $\begin{array}{l}\text { Berry et al. } \\
2004^{43}\end{array}$ & $\begin{array}{l}\text { To compare } \\
\text { doctors' and lay } \\
\text { people's } \\
\text { interpretation of } \\
\text { the EC verbal } \\
\text { descriptors }\end{array}$ & $\begin{array}{l}\text { Numerical } \\
\text { estimates }\end{array}$ & 134 & $\begin{array}{l}\text { Undergraduate } \\
\text { and postgraduate } \\
\text { students }\end{array}$ & $\begin{array}{l}\text { Medication } \\
\text { side effect }\end{array}$ & No & Yes & $\begin{array}{l}\text { A little } \\
\text { concern }\end{array}$ \\
\hline $\begin{array}{l}\text { Knapp et al. } \\
2004^{44}\end{array}$ & $\begin{array}{l}\text { To explore whether } \\
\text { the EC verbal } \\
\text { descriptors } \\
\text { effectively } \\
\text { convey the risk of } \\
\text { side effects }\end{array}$ & $\begin{array}{l}\text { Numerical } \\
\text { estimates }\end{array}$ & 120 & $\begin{array}{l}\text { Adults from } \\
\text { cardiac } \\
\text { rehabilitation } \\
\text { clinics following } \\
\text { a recent } \\
\text { admission }\end{array}$ & $\begin{array}{l}\text { Medication } \\
\text { side effects for } \\
\text { cardiac } \\
\text { medication }\end{array}$ & No & Yes & $\begin{array}{l}\text { A little } \\
\text { concern }\end{array}$ \\
\hline $\begin{array}{l}\text { Berry and } \\
\text { Hochhauser } \\
2006^{45}\end{array}$ & $\begin{array}{l}\text { To assess how } \\
\text { verbal descriptors } \\
\text { affect people's } \\
\text { perceptions } \\
\text { of clinical trial } \\
\text { participation risks }\end{array}$ & $\begin{array}{l}\text { Numerical } \\
\text { estimates }\end{array}$ & 96 & $\begin{array}{l}\text { Adults from a } \\
\text { train station }\end{array}$ & $\begin{array}{l}\text { Fictional } \\
\text { serious skin } \\
\text { condition }\end{array}$ & No & No & $\begin{array}{l}\text { A little } \\
\text { concern }\end{array}$ \\
\hline $\begin{array}{l}\text { Hubal and } \\
\text { Day } 2006^{46}\end{array}$ & $\begin{array}{l}\text { To evaluate the } \\
\text { understanding of } \\
\text { verbal probability } \\
\text { terms } \\
\text { and effects of } \\
\text { alternative formats }\end{array}$ & $\begin{array}{l}\text { Numerical } \\
\text { estimates }\end{array}$ & 222 & $\begin{array}{l}\text { Undergraduate } \\
\text { students }\end{array}$ & $\begin{array}{l}\text { Medication } \\
\text { side effect }\end{array}$ & No & No & $\begin{array}{l}\text { Moderate } \\
\text { concern }\end{array}$ \\
\hline $\begin{array}{l}\text { Young and } \\
\text { Oppenheimer } \\
2006^{47}\end{array}$ & $\begin{array}{l}\text { To assess how } \\
\text { different formats of } \\
\text { risk information } \\
\text { influence } \\
\text { medication } \\
\text { compliance }\end{array}$ & $\begin{array}{l}\text { Numerical } \\
\text { estimates }\end{array}$ & 120 & $\begin{array}{l}\text { Adult students } \\
\text { from a university }\end{array}$ & $\begin{array}{l}\text { Medication } \\
\text { side effect }\end{array}$ & No & Yes & $\begin{array}{l}\text { Moderate } \\
\text { concern }\end{array}$ \\
\hline $\begin{array}{l}\text { France et al. } \\
2008^{48}\end{array}$ & $\begin{array}{l}\text { To compare the } \\
\text { understanding of } \\
\text { frequency of side } \\
\text { effects } \\
\text { when expressed in } \\
\text { percentages or } \\
\text { descriptive } \\
\text { language }\end{array}$ & $\begin{array}{l}\text { Numerical } \\
\text { estimates, } \\
\% \text { correctly } \\
\text { identified }\end{array}$ & 50 & $\begin{array}{l}\text { Patients in the } \\
\text { chest pain unit of } \\
\text { an urban } \\
\text { emergency } \\
\text { department who } \\
\text { had one or more } \\
\text { ischemic heart } \\
\text { disease factors }\end{array}$ & $\begin{array}{l}\text { Risks of } \\
\text { treatment for } \\
\text { acute } \\
\text { myocardial } \\
\text { infarction }\end{array}$ & $\begin{array}{l}\text { Severe vs less } \\
\text { severe side } \\
\text { effects }\end{array}$ & No & No concern \\
\hline $\begin{array}{l}\text { Graham et al. } \\
2009^{49}\end{array}$ & $\begin{array}{l}\text { To identify } \\
\text { women's } \\
\text { preference and } \\
\text { interpretation of } \\
\text { language } \\
\text { for description of } \\
\text { the size of }\end{array}$ & $\begin{array}{l}\text { Format } \\
\text { preference }\end{array}$ & 262 & $\begin{array}{l}\text { Adult female } \\
\text { patients } \\
\text { undergoing } \\
\text { routine follow-up } \\
\text { visits for breast } \\
\text { cancer }\end{array}$ & $\begin{array}{l}\text { Breast cancer } \\
\text { risk }\end{array}$ & No & No & $\begin{array}{l}\text { A little } \\
\text { concern }\end{array}$ \\
\hline
\end{tabular}


Table 1. (continued)

\begin{tabular}{|c|c|c|c|c|c|c|c|c|}
\hline $\begin{array}{l}\text { Authors and } \\
\text { year }\end{array}$ & Research question & $\begin{array}{l}\text { Primary } \\
\text { outcome } \\
\text { measured }\end{array}$ & $\begin{array}{l}\text { Sample } \\
\text { size* }\end{array}$ & $\begin{array}{l}\text { Sample } \\
\text { description* }\end{array}$ & $\begin{array}{l}\text { Health } \\
\text { condition or } \\
\text { situation }\end{array}$ & $\begin{array}{l}\text { Severity of } \\
\text { health event } \\
\text { specified? }\end{array}$ & $\begin{array}{l}\text { Study } \\
\text { of EC } \dagger \\
\text { terms? }\end{array}$ & $\begin{array}{l}\text { Risk of } \\
\text { bias } \\
\text { assessment }\end{array}$ \\
\hline $\begin{array}{l}\text { Knapp et al. } \\
2009^{50}\end{array}$ & $\begin{array}{l}\text { treatment } \\
\text { complication risks } \\
\text { To assess the } \\
\text { effectiveness of } \\
\text { presenting side } \\
\text { effect risk } \\
\text { information in } \\
\text { different formats }\end{array}$ & $\begin{array}{l}\text { Numerical } \\
\text { estimates }\end{array}$ & 148 & $\begin{array}{l}\text { Adult users of an } \\
\text { online cancer } \\
\text { information } \\
\text { website }\end{array}$ & $\begin{array}{l}\text { Medication } \\
\text { side effect }\end{array}$ & No & No & No concern \\
\hline $\begin{array}{l}\text { Nagle et al. } \\
2009^{51}\end{array}$ & $\begin{array}{l}\text { To evaluate female } \\
\text { patients' preference } \\
\text { on risk of disease }\end{array}$ & $\begin{array}{l}\text { Format } \\
\text { preference }\end{array}$ & 294 & $\begin{array}{l}\text { Adult female } \\
\text { patients from a } \\
\text { maternity unit }\end{array}$ & $\begin{array}{l}\text { Down } \\
\text { syndrome risk }\end{array}$ & No & No & No concern \\
\hline $\begin{array}{l}\text { Cheung et al. } \\
2010^{52}\end{array}$ & $\begin{array}{l}\text { To compare } \\
\text { patients' preference } \\
\text { for risk } \\
\text { presentation in } \\
\text { medications }\end{array}$ & $\begin{array}{l}\text { Format } \\
\text { preference }\end{array}$ & 240 & $\begin{array}{l}\text { Adult patients } \\
\text { from arthritis } \\
\text { clinics in a } \\
\text { hospital and } \\
\text { outpatient } \\
\text { practice }\end{array}$ & $\begin{array}{l}\text { Pain relief } \\
\text { medication }\end{array}$ & No & Yes & $\begin{array}{l}\text { A little } \\
\text { concern }\end{array}$ \\
\hline Vahabi $2010^{53}$ & $\begin{array}{l}\text { To evaluate } \\
\text { whether format } \\
\text { preference } \\
\text { influences } \\
\text { comprehension }\end{array}$ & $\begin{array}{l}\text { Format } \\
\text { preference }\end{array}$ & 180 & $\begin{array}{l}\text { Adult female } \\
\text { patients from } \\
\text { various } \\
\text { community } \\
\text { settings }\end{array}$ & $\begin{array}{l}\text { Breast cancer } \\
\text { risk }\end{array}$ & No & No & $\begin{array}{l}\text { High } \\
\text { concern }\end{array}$ \\
\hline $\begin{array}{l}\text { Peters et al. } \\
2014^{54}\end{array}$ & $\begin{array}{l}\text { To measure risk } \\
\text { comprehension and } \\
\text { willingness to use a } \\
\text { medication } \\
\text { when presented } \\
\text { with different } \\
\text { formats }\end{array}$ & $\begin{array}{l}\text { Numerical } \\
\text { estimates }\end{array}$ & 905 & $\begin{array}{l}\text { Adult participants } \\
\text { from a paid } \\
\text { online } \\
\text { questionnaire }\end{array}$ & $\begin{array}{l}\text { Cholesterol } \\
\text { medication }\end{array}$ & No & Yes & No concern \\
\hline $\begin{array}{l}\text { Knapp et al. } \\
2014^{55}\end{array}$ & $\begin{array}{l}\text { To evaluate } \\
\text { recommendations } \\
\text { on communicating } \\
\text { frequency } \\
\text { information on side } \\
\text { effect risk }\end{array}$ & $\begin{array}{l}\text { Numerical } \\
\text { estimates }\end{array}$ & 339 & $\begin{array}{l}\text { Adult users of an } \\
\text { online cancer } \\
\text { information } \\
\text { website }\end{array}$ & $\begin{array}{l}\text { Medication } \\
\text { side effects }\end{array}$ & No & No & $\begin{array}{l}\text { A little } \\
\text { concern }\end{array}$ \\
\hline $\begin{array}{l}\text { Webster et al. } \\
2017^{56}\end{array}$ & $\begin{array}{l}\text { To assess how } \\
\text { people interpret the } \\
\text { EC verbal } \\
\text { descriptors }\end{array}$ & $\begin{array}{l}\text { Numerical } \\
\text { estimates, } \\
\% \text { correctly } \\
\text { identified }\end{array}$ & 1003 & $\begin{array}{l}\text { Adult users of an } \\
\text { online survey } \\
\text { conducted by a } \\
\text { market research } \\
\text { company }\end{array}$ & $\begin{array}{l}\text { Medication } \\
\text { side effects }\end{array}$ & $\begin{array}{l}\text { Mild vs severe } \\
\text { side effects }\end{array}$ & Yes & $\begin{array}{l}\text { A little } \\
\text { concern }\end{array}$ \\
\hline $\begin{array}{l}\text { Carey et al. } \\
2018\end{array}$ & $\begin{array}{l}\text { To assess patients' } \\
\text { interpretation of } \\
\text { verbal descriptor } \\
\text { chance } \\
\text { of remission and } \\
\text { preferences for } \\
\text { format of risk } \\
\text { communication }\end{array}$ & $\begin{array}{l}\text { Numerical } \\
\text { estimates, } \\
\text { format } \\
\text { preference }\end{array}$ & 210 & $\begin{array}{l}\text { Adult medical } \\
\text { oncology } \\
\text { outpatients with a } \\
\text { diagnosis of } \\
\text { cancer }\end{array}$ & $\begin{array}{l}\text { Cancer long- } \\
\text { term side ef- } \\
\text { fects and } \\
\text { chances of re- } \\
\text { mission }\end{array}$ & No & No & $\begin{array}{l}\text { A little } \\
\text { concern }\end{array}$ \\
\hline $\begin{array}{l}\text { Wiles et al. } \\
2020^{58}\end{array}$ & $\begin{array}{l}\text { To determine the } \\
\text { perceived risk of } \\
\text { surgical } \\
\text { complication } \\
\text { risk using verbal } \\
\text { probability terms }\end{array}$ & $\begin{array}{l}\text { Numerical } \\
\text { estimates }\end{array}$ & 290 & $\begin{array}{l}\text { Adult patients } \\
\text { attending a pre- } \\
\text { operative assess- } \\
\text { ment in a clinic }\end{array}$ & $\begin{array}{l}\text { Major adverse } \\
\text { postoperative } \\
\text { complication }\end{array}$ & No & No & No concern \\
\hline
\end{tabular}

*Several studies contained both subsamples that met our inclusion criteria (adult laypeople) and other subsamples that did not (physicians, adolescents). As described in the "METHODS" section, these studies were included if the results for the eligible subsample were reported separately. For these studies, we report the sample size and sample description of the subgroup that met our inclusion criteria

$\dagger E C=$ European Community

tGraham et al. $2009^{49}$ required respondents to choose from ordinal categories ranging from 1/100 to 1/10 000. The modal interpretation of "sometimes" was 1/100 (36\% of women), "uncommon" 1/1000 (35\%), "very uncommon" $1 / 10000$ (40\%), "rare" $1 / 10000$ (58\%) and "very rare" 11 10000 (51\%). Because of the categorical assessment and the fact that no larger numbers were provided to choose from, we did not average these results into the findings in Table 2.

Australia, 1 in Canada, and 1 in Singapore. Fifteen focused on medication side effects, 14 on disease risks, and 4 with no specified context. Many studies provided little demographic information, with only 27 reporting participant gender, 25 age, 24 education, 5 ethnicity, and 4 socioeconomic status. Fourteen studies included actual patients, although primarily in hypothetical scenarios, while others recruited students, or members from the public (column 5 in Table 1). Most studies used relatively simple questionnaire study designs, and as a result most 
$(64 \%, n=21)$ had low or no risk of bias; 2 had high risk of bias.

Below, we present findings from the 2 subsets, which are not mutually exclusive: (1) 24 studies that elicited numerical estimates for verbal probability terms, including 9 focusing specifically on the EC terms (Table 2; Fig. 2), and (2) 11 studies (one of which contained 2 different samples) that assessed preferences for verbal versus quantitative risk information (Table 3).

Subset 1:

In 24 studies, numerical estimates were elicited for verbal probability terms. A total of 145 unique verbal probability terms were studied (Appendix 2). In some studies, the researchers also specified the severity of the event described by the verbal probability. We considered it likely that probability of mild outcome might be perceived differently than probability of a severe one. Therefore, we present these conditions separately, resulting in 14 unique probability-severity combinations (Table 2). Table 2 and Appendix 3 report pooled averages and ranges for 14 terms that were evaluated in at least three studies each and reported sufficient information for the meta-analysis. The term "rare" was estimated to mean a $10 \%$ risk, whereas the term "very likely" averaged $84 \%$. Variability of interpretation of these terms was high, both across studies (minimum and maximum study averages reported in Table 2 column 5 and 6) and within study (ranges reported in column 7). For example, individuals estimated the term "rare" to mean anything from 0 to $80 \%$, and "common" to mean anywhere between 10 and $100 \%$. The effect of specifying the severity of the health event was modest. A "rare severe" event was judged slightly less likely than a "rare mild" event $(10.1 \%$ versus $14.1 \%$ respectively), and a "common severe" event as slightly less likely than a "common mild" one ( $43.1 \%$ versus $50.5 \%)$. A subset of studies ( 9 indicated in Table 1) specifically examined interpretations of the EC probability labels. Meaningful summary data could not be generated for participant type (university student vs other adults) or information type (medication side effect vs procedure side effect vs disease risk)

because samples in these subgroups were too small.

In 2 of these studies, researchers additionally evaluated whether the participants misinterpreted the verbal probability term, defining misinterpretation as an estimate differing from the EC definition of the term (Fig. 2). As shown in Fig. 2, misinterpretation rates were higher for the terms indicating rare events, and there was relatively little difference between misinterpretation of the chances of mild events, severe events, and events of unspecified severity (Fig. 2).

Subset 2:

In 11 studies, participants' preference for verbal versus numeric information was captured (one study contained 2 independently recruited samples for a total of 12 samples: Table 3). In 10 of the 12 samples, majorities (proportions ranging from 54 to $95 \%$ ) preferred numeric risk information alone or in combination with verbal labels. In the 6 samples that had a choice between verbal, numeric, and combined formats, from 18 to $54 \%$ of respondents preferred the combination of numeric with verbal descriptions.

\section{DISCUSSION}

Since 1967, 33 studies have examined lay interpretation of and preferences for verbal probability terms such as "rare" and "common" in health and medical contexts. These studies show that lay peoples' numeric interpretations of these verbal terms are extremely variable and highly overlapping. For example, across the studies, individual participants estimated the term "rare" at anywhere between 0 and $80 \%$ probability, and the term "common" at between 10 and $100 \%$ probability. In other words, these studies provide no assurance that patients will perceive a health outcome described as "common" as more likely than one described as

Table 2 Numeric Estimates of Verbal Probability Terms

\begin{tabular}{|c|c|c|c|c|c|c|}
\hline Verbal probability term & $\begin{array}{l}\text { Number } \\
\text { of studies }\end{array}$ & $\begin{array}{l}\text { Average numeric } \\
\text { estimate, random } \\
\text { effects model }(\%)\end{array}$ & $95 \%$ CI (\%) & $\begin{array}{l}\text { Minimum } \\
\text { sample average } \\
(\%)^{*}\end{array}$ & $\begin{array}{l}\text { Maximum } \\
\text { sample average } \\
(\%)^{*}\end{array}$ & $\begin{array}{l}\text { Range of individual } \\
\text { estimates }(\%)^{\dagger}\end{array}$ \\
\hline Rare(ly) & 7 & 10.00 & {$[7.99,12.01]$} & 7.0 & 21 & $0-80$ \\
\hline Rare-severe event & 3 & 10.06 & {$[5.45,14.68]$} & 6.3 & 34.8 & - \\
\hline Rare-mild event & 3 & 14.14 & {$[7.88,20.40]$} & 9.6 & 39.3 & - \\
\hline Uncommon & 4 & 17.64 & {$[13.19,22.09]$} & 13.3 & 22.9 & $0-90$ \\
\hline Unlikely & 6 & 17.71 & {$[14.86,20.55]$} & 13.3 & 27 & $0-85$ \\
\hline Common-severe event & 3 & 43.08 & {$[40.27,45.88]$} & 41.9 & 45.6 & - \\
\hline Possible(ly) & 6 & 43.28 & {$[36.66,49.89]$} & 36.9 & 62 & - \\
\hline Common-mild event & 3 & 50.47 & {$[45.59,55.34]$} & 48 & 58 & - \\
\hline Common & 6 & 58.73 & {$[50.40,67.06]$} & 34.2 & 70.5 & $10-100$ \\
\hline Very common & 3 & 60.10 & {$[42.36,77.85]$} & 38.5 & 71.6 & $5-100$ \\
\hline Probable(ly) & 5 & 69.87 & {$[67.07,72.67]$} & 66 & 73.9 & $20-100$ \\
\hline Likely & 6 & 71.87 & {$[69.90,73.84]$} & 66 & 94 & - \\
\hline Usual(ly) & 3 & 75.38 & {$[71.53,79.23]$} & 72 & 78 & - \\
\hline Very likely & 3 & 84.30 & {$[79.43,89.17]$} & 75.2 & 93 & $20-100$ \\
\hline
\end{tabular}

Table includes terms studied in 3 or more studies in which sufficient information was reported for the meta-analysis

*All 19 studies reported an average estimate; minimum is the lowest of these averages, and maximum is the highest

tOnly 4 studies reported ranges of estimates provided by individual participants within the study. This column reflects the range across all 4 studies 
Average proportion misinterpreting EC risk labels

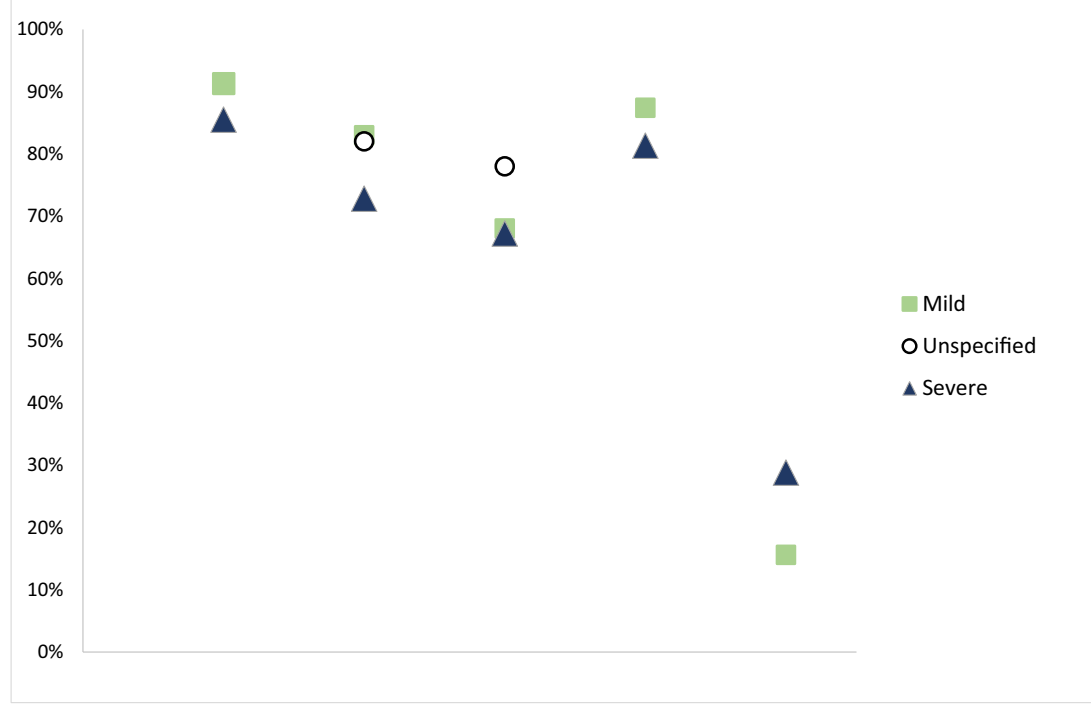

Figure 2 Average proportions misinterpreting European Commission (EC) risk labels across 2 studies. Legend: Among 2 large studies of EC verbal labels, including 1053 participants, an average of $70.1 \%$ misinterpreted the EC risk label. Rates of misinterpretation were similar whether the severity of the event was described or not, and if it was described, whether it was "mild" or "severe." Misinterpretations were more common for more rare events, and there were only modest differences between interpretation of events described as "severe" versus "mild."

"rare." This suggests that providers and health communicators should provide numbers where possible, avoiding situations in which words alone are used to describe risk.

In addition, the subset of studies examining the European Commission (EC) verbal terminology for risk in drug labels shows that these terms are usually misinterpreted and lead to numeric estimates far higher than the developers intended. For example, in the EC terminology, "rare" is intended to describe a risk between 0.1 and $0.01 \%$, but the average lay interpretation was almost $10 \%$, more than 100-fold higher. The EC term "common," meant to describe a risk between 1 and 10\%, was interpreted as an average of about 59\%. It is clear that this verbal risk terminology is miscalibrated to lay perceptions, particularly for rare events. In particular, providers and health communicators who use the EC terms to describe chance of medication side effects should recognize that these terms are likely to vastly inflate perceptions of side effect risk.

The literature also suggests that majorities of patients prefer numeric risk information, alone or in combination with verbal labels. This finding suggests that healthcare professionals who choose verbal-only risk descriptors may not be meeting the preferences of their patients.

Overall, findings of these studies about health and medical risk communication are congruent with risk communication research in non-medical domains, which has similarly outlined the variability in interpretations of these terms. ${ }^{10-13}$

One limitation of our study stems from the search approach. It was challenging to create a literature search strategy for this problem because of the ubiquity of terms such as "risk" in non-communication domains such as epidemiology. We

Table 3 Numbers and Proportions Preferring Verbal or Numeric Probabilities

\begin{tabular}{|c|c|c|c|c|c|}
\hline \multirow[t]{2}{*}{ Study * } & \multirow[t]{2}{*}{ Sample size } & \multicolumn{4}{|l|}{$n(\%)$} \\
\hline & & Preferred verbal & Preferred numeric & Preferred combination & No preference \\
\hline Woloshin et al. $1984^{25}$ & 307 & $91(29.6)$ & $135(43.9)$ & $81(26.3)$ & NA \\
\hline Shaw and Dear $1990^{23}$ & 81 & $43(53.1)$ & $30(37.0)$ & NA & $8(9.9)$ \\
\hline Freeman and Bass $1992^{6}$ & 208 & $89(42.7)$ & $119(57.2)$ & NA & $\mathrm{NA}$ \\
\hline Hallowell et al. $19977^{33}$ & 43 & $3(7)$ & $9(21)$ & $22(52)$ & $8(19)$ \\
\hline Franic and Pathak $2000^{26}$ & 74 & $4(5.4)$ & $70(94.6)$ & NA & NA \\
\hline \multirow[t]{2}{*}{ Lobb et al. $2003^{41}$} & 109 (unaffected by condition) & $24(22.1)$ & $55(50)$ & $20(18.3)$ & $10(9.6)$ \\
\hline & 84 (affected by condition) & $15(17.9)$ & $16(19.2)$ & $45(53.8)$ & $8(9)$ \\
\hline Graham et al. $2009^{49}$ & 262 & $136(52)$ & $125(47.7)$ & NA & $1(0.3)$ \\
\hline Nagle et al. $2009^{38}$ & 294 & $85(28.9)$ & $132(44.9)$ & $76(25.8)$ & NA \\
\hline Cheung et al. $2010^{39}$ & 240 & $60(25.0)$ & $180(75.0)$ & NA & NA \\
\hline Vahabi $2010^{40}$ & 180 & $61(33.9)$ & $119(66.1)$ & NA & NA \\
\hline Carey et al. $2018^{57}$ & 210 & $59(28)$ & $33(16)$ & $79(38)$ & 39 (18) \\
\hline
\end{tabular}

NA indicates that this option was not presented to respondents

*Kaplowitz et al. (2002) ${ }^{36}$ also evaluated format preference among cancer patients and survivors in a hypothetical choice between a verbal probability and a quantitative estimate of survival. However, the findings are not integrated into this table because the options were not mutually exclusive, and the authors do not clarify how many patients chose both. (Table 1 of that paper shows that $80 \%$ endorsed verbal probabilities and $53 \%$ endorsed quantitative information, suggesting that some subset must have chosen both) 
ended up calibrating the search strategy fairly broadly, requiring manual review to narrow down eligible articles. We did not restrict the search to a specific date range, and it is possible that language interpretation has changed over time. Another limitation is in the completeness of the research studies included, many of which did not provide details of demographics. We are therefore unable to draw conclusions from this review about the effects of education level, literacy, numeracy, socioeconomic status, race, or ethnicity on interpretations and information preferences. Other research has demonstrated that preference for numeric information is stronger among those with higher education or numeracy, and that individuals with lower levels of numeracy may express less comfort with numeric risk information. ${ }^{59}$ In addition, it is likely that numeracy would influence patients' ability to assign a numeric probability to a verbal term. ${ }^{3,60}$ In focusing on the contrast between verbal and numeric risk information, we did not examine the vast literature on visualization and risk communication.

In summary, a systematic review of the literature provides strong evidence that patient interpretations of verbal probability terms are so variable that they may not distinguish between events of very different likelihoods. The evidence also suggests that most patients prefer numeric information about risks, either alone or in combination with verbal labels. These findings suggest that health professionals who avoid numbers by providing verbal probabilities alone are likely to have poor communication with their patients. Physicians and other healthcare professionals can improve the effectiveness of their communication with patients by providing accurate quantitative information about health risks.

Acknowledgements: This study was funded by the National Library of Medicine (RO1 LMO12964, PI Ancker). The authors gratefully acknowledge the discussion and input by the Making Numbers Meaningful Numeracy Expert Panel.

Corresponding Author: Jessica S Ancker, MPH, PhD; Department of Biomedical Informatics, Vanderbilt University Medical Center, Nashville, TN, USA (e-mail: jessica.s.ancker@vumc.org).

Supplementary Information The online version contains supplementary material available at https://doi.org/10.1007/s11606-02107050-7.

\section{Declarations:}

Conflict of Interest: All authors report they had no competing interests to declare.

Open Access This article is licensed under a Creative Commons Attribution 4.0 International License, which permits use, sharing, adaptation, distribution and reproduction in any medium or format, as long as you give appropriate credit to the original author(s) and the source, provide a link to the Creative Commons licence, and indicate if changes were made. The images or other third party material in this article are included in the article's Creative Commons licence, unless indicated otherwise in a credit line to the material. If material is not included in the article's Creative Commons licence and your intended use is not permitted by statutory regulation or exceeds the permitted use, you will need to obtain permission directly from the copyright holder. To view a copy of this licence, visit http://creativecommons. org/licenses/by/4.0/.

\section{REFERENCES}

1. Lipkus IM, Samsa G, Rimer BK. General performance on a numeracy scale among highly educated samples. Medical Decision Making. 2001;21:37-44.

2. Peters E, Vastfjall D, Slovic P, Mertz CK, Mazzocco K, Dickert S. Numeracy and decision making. Psychological Science. 2006;17(5):407413.

3. Ancker JS, Kaufman D. Rethinking health numeracy: a multidisciplinary literature review. Journal of the American Medical Informatics Association : JAMIA. 2007;14(6):713-721. doi:https://doi.org/10.1197/jamia.M2464

4. Reyna VF, Nelson WL, Han P, Dieckmann N. How numeracy influences risk comprehension and medical decision-making. Psychological Bulletin. 2009; 135(6):943-973.

5. Peters E, Hart PS, Fraenkel L. Informing patients: the influence of numeracy, framing, and format of side effect information on risk perceptions. Medical Decision Making. 2011;31:432-436.

6. Freeman TR, Bass MJ. Risk language preferred by mothers in considering a hypothetical new vaccine for their children. CMAJ : Canadian Medical Association journal = journal de l'Association medicale canadienne. 1992;147(7):1013-1017.

7. Anderson BL, Obrecht NA, Chapman GB, Driscoll DA, Schulkin J. Physicians' communication of Down syndrome screening test results: the influence of physician numeracy. Genetics in Medicine. 2011;13(8):744749 https://doi.org/10.1097/GIM.0b013e31821a370f

8. Neuner-Jehle S, Senn O, Wegwarth O, Rosemann T, Steurer J. How do family physicians communicate about cardiovascular risk? Frequencies and determinants of different communication formats. BMC Family Practice. 2011;12(1):15. doi:https://doi.org/10.1186/1471-2296-12-15

9. Petrova D, Kostopoulou O, Delaney BC, Cokely ET, Garcia-Retamero R. Strengths and gaps in physicians' risk communication: a scenario study of the influence of numeracy on cancer screening communication. Med Decis Making. 2018;38(3):355-365. doi:https://doi.org/10.1177/ 0272989x17729359

10. Brun W, Teigen, K. H. Verbal probabilities: Ambiguous, context-dependent, or both? Organizational Behavior and Human Decision Processes. 1988;41:390-404.

11. Wintle BC, Fraser H, Wills BC, Nicholson AE, Fidler F. Verbal probabilities: Very likely to be somewhat more confusing than numbers. PLOS One. 2019;14(4):e0213522. doi:https://doi.org/10.1371/journal.pone. 0213522

12. Karelitz TM, Budescu DV. You say "probable" and I say "likely": improving interpersonal communication with verbal probability phrases. $J$ Exp Psychol Appl. 2004;10(1):25-41. doi:https://doi.org/10.1037/1076898x.10.1.25

13. Visschers VHM, Meertens RM, Passchier WWF, De Vries NNK. Probability information in risk communication: a review of the research literature. Risk Analysis. 2009;29(2):267-287. doi:https://doi.org/10.1111/j.15396924.2008.01137.x

14. Juanchich M, Teigen KH, Villejoubert G. Is guilt 'likely' or 'not certain'? Contrast with previous probabilities determines choice of verbal terms. Acta Psychol (Amst). 2010;135(3):267-77. doi:https://doi.org/10.1016/j. actpsy.2010.04.016

15. Juanchich M, Sirota M. Do people really say it is "likely" when they believe it is only "possible"? Effect of politeness on risk communication. Quarterly journal of experimental psychology (2006). 2013;66(7):1268-75. doi: 10.1080/17470218.2013.804582

16. Weber EU, Blais AR, Betz N. A domain-specific risk-attitude scale: measuring risk perceptions and risk behaviors. Journal of Behavioral Decision Making. 2002;(15):1-28.

17. Liberati A, Altman D, Tetzlaff J, et alThe PRISMA statement for reporting systematic reviews and meta-analyses of studies that evaluate healthcare interventions: explanation and elaboration. BMJ; 2009.

18. Pharmaceutical Committee EC. A guideline on the readability of the label and package leaflet of medicinal products for human use. 1998. https:// ec.europa.eu/health/sites/default/files/files/eudralex/vol-2/c/2009_ 01_12_readability_guideline_final_en.pdf. Accessed 30 Jul 2021

19. Riley RD, Higgins JPT, Deeks JJ. Interpretation of random effects metaanalyses. BMJ. 2011;342:d549. doi:https://doi.org/10.1136/bmj.d549 
20. Fleiss JL. The statistical basis of meta-analysis. Stat Methods Med Res. 1993;2(2):121-45. doi:https://doi.org/10.1177/096228029300200202

21. DerSimonian R, Laird N. Meta-analysis in clinical trials revisited. Contemporary clinical trials. 2015;45(Pt A):139-145. https://doi.org/10. 1016/j.cct.2015.09.002

22. Jackson D, Bowden J. Confidence intervals for the between-study variance in random-effects meta-analysis using generalised heterogeneity statistics: should we use unequal tails? BMC Medical Research Methodology. 2016;16(1):118. https://doi.org/10.1186/s12874-016-0219-y

23. R Core Team. $R$ : A language and environment for statistical computing. $\mathrm{R}$ Foundation for Statistical Computing, 2021.

24. Balduzzi S, Rücker G, Schwarzer G. How to perform a meta-analysis with R: a practical tutorial. Evidence Based Mental Health. 2019;22(4):153 160. doi:https://doi.org/10.1136/ebmental-2019-300117

25. Quality AfHRa. Methods guide for effectiveness and comparative effectiveness reviews.

26. al JPTHe. The Cochrane Collaboration's tool for assessing risk of bias in randomised trials. BMJ 2011. p. d5928.

27. Lichtenstein S, Newman JR. Empirical scaling of common verbal phrases associated with numerical probabilities. Psychonomic Science. 1967;9:563-564.

28. Budescu DV, Wallsten TS. Consistency in interpretation of probabilistic phrases. Organizational Behavior and Human Decision Processes. 1985;36(3):391-405. doi:https://doi.org/10.1016/0749-5978(85)90007$\mathrm{X}$

29. Reagan RT, Mosteller F, Youtz C. Quantitative meanings of verbal probability expressions. J Appl Psychol. Jun 1989;74(3):433-42. doi:https://doi.org/10.1037/0021-9010.74.3.433

30. Shaw NJ, Dear PR. How do parents of babies interpret qualitative expressions of probability? Arch Dis Child. May 1990;65(5):520-3. doi:https://doi.org/10.1136/adc.65.5.520

31. Weber E, Hilton D. Contextual effects in the interpretations of probability words: perceived base rate and severity of events. Journal of Experimental Psychology: Human Perception and Performance. 1990;16 https://doi org/10.1037/0096-1523.16.4.781

32. Woloshin KK, Ruffin MTt, Gorenflo DW. Patients' interpretation of qualitative probability statements. Arch Fam Med. 1994;3(11):961-6. doi:https://doi.org/10.1001/archfami.3.11.961

33. Hallowell NS, Helen Murton, Frances Green, Jo, Richards M. "Talking about chance": the presentation of risk information during genetic counseling for breast and ovarian cancer. Journal of Genetic Counseling. 1997;6(3)

34. Franic DM, Pathak DS. Communicating the frequency of adverse drug reactions to female patients. Drug Information Journal. 2000;34(1):251272. doi:https://doi.org/10.1177/009286150003400134

35. Biehl M. Halpern-Felsher BL. Adolescents' and adults' understanding of probability expressions. J Adolesc Health. Jan 2001;28(1):30-5. doi:https://doi.org/10.1016/s1054-139x(00)00176-2

36. Kaplowitz SA, Campo S, Chiu WT. Cancer patients' desires for communication of prognosis information. Health Commun. 2002;14(2):221-41. doi:https://doi.org/10.1207/S15327027HC1402_4

37. Berry DC, Knapp PR, Raynor T. Is 15 per cent very common? Informing people about the risks of medication side effects. International Journal of Pharmacy Practice. 2002;10(3):145-151. doi:https://doi.org/10.1111/j. 2042-7174.2002.tb00602.x

38. Berry D, Raynor D, Knapp P. Communicating risk of medication side effects: an empirical evaluation of EU recommended terminology. Psychology, Health and Medicine. 2003;8:251-263. https://doi.org/10. 1080/1354850031000135704

39. Budescu DV, Karelitz TM, Wallsten TS. Predicting the directionality of probability words from their membership functions. Journal of Behavioral Decision Making. 2003;16(3):159-180. doi:https://doi.org/10.1002/ bdm. 440

40. Davey HM, Lim J, Butow PN, Barratt AL, Houssami N, Higginson R. Consumer information materials for diagnostic breast tests: women's views on information and their understanding of test results. Health Expect. 2003;6(4):298-311. doi:https://doi.org/10.1046/j.1369-7625. 2003.00227.x

41. Lobb EA, P N Meiser, B Barratt, A Gaff, C Young, M A Kirk, J Gattas, MM Gleeson , Tucker K. Women's preferences and consultants' communication of risk in consultations about familial breast cancer: impact on patient outcomes. J Med Genet. 2003;40(56)

42. Berry D, Raynor T, Knapp P, Bersellini E. Over the counter medicines and the need for immediate action: a further evaluation of European Commission recommended wordings for communicating risk. Patient
Educ Couns. 2004;53(2):129-34. doi:https://doi.org/10.1016/s07383991(03)00111-3

43. Berry DC, Holden W, Bersellini E. Interpretation of recommended risk terms: differences between doctors and lay people. International Journal of Pharmacy Practice. 2004;12(3):117-124. doi:https://doi.org/10.1211/ 0022357044120

44. Knapp P, Raynor DK, Berry DC. Comparison of two methods of presenting risk information to patients about the side effects of medicines. Quality \& safety in health care. 2004;13(3):176-180. https://doi. org/10.1136/qhc. 13.3 .176

45. Berry DC, Hochhauser M. Verbal labels can triple perceived risk in clinical trials. Drug Information Journal. 2006;40(3):249-258. doi:https:// doi.org/10.1177/009286150604000302

46. Rob Hubal RSD. Understanding the frequency and severity of side effects: linguistic, numeric, and visual representations. American Association for Artificial Intelligence 2006;

47. Young SD, Oppenheimer DM. Different methods of presenting risk information and their influence on medication compliance intentions: results of three studies. Clin Ther. 2006;28(1):129-39. doi:https://doi. org/10.1016/j.clinthera.2006.01.013

48. France J, Keen C, Bowyer S. Communicating risk to emergency department patients with chest pain. Emerg Med J. 2008;25(5):276-8. doi:https://doi.org/10.1136/emj.2007.054106

49. GRAHAM PH, MARTIN RM, BROWNE LH. Communicating breast cancer treatment complication risks: when words are likely to fail ajco_1232 193..199. Asia-Pacific Journal of Clinical Oncology. 2009;5:193-199.

50. Knapp P, Gardner PH, Carrigan N, Raynor DK, Woolf E. Perceived risk of medicine side effects in users of a patient information website: a study of the use of verbal descriptors, percentages and natural frequencies. British journal of health psychology. 2009;14(Pt 3):579-94. https://doi.org/10. 1348/135910708x375344

51. Nagle C, Hodges R, Wolfe R, Wallace E. Reporting down syndrome screening results: women's understanding of risk. Prenatal diagnosis. 2009;29:234-9. https://doi.org/10.1002/pd.2210

52. Cheung Y, Wee H, Thumboo J, et al. Risk communication in clinical trials: a cognitive experiment and a survey. BMC medical informatics and decision making. 2010;10:55. https://doi.org/10.1186/1472-6947-1055

53. Vahabi M. Verbal versus numerical probabilities: Does format presentation of probabilistic information regarding breast cancer screening affect women's comprehension? Health Education Journal. 2010;69(2): 150-163. doi:https://doi.org/10.1177/0017896909349262

54. Peters E, Hart PS, Tusler M, Fraenkel L. Numbers matter to informed patient choices: a randomized design across age and numeracy levels. Medical decision making : an international journal of the Society for Medical Decision Making. 2014;34(4):430-442. doi:https://doi.org/10. 1177/0272989X13511705

55. Knapp P, Gardner P, Woolf E. Combined verbal and numerical expressions increase perceived risk of medicine side-effects: a randomized controlled trial of EMA recommendations. Health Expectations; 2014. p. pp. 264-274.

56. Webster R, Weinman J, Rubin J. People's understanding of verbal risk descriptors in patient information leaflets: a cross-sectional national survey of 18- to 65-Year-Olds in England. Drug safety: an international journal of medical toxicology and drug experience.2017. p. 743-754.

57. Carey M, Herrmann A, Hall A, K Mef. Exploring health literacy and preferences for risk communication among medical oncologypatients. PLOS ONE. 2018;13(9)

58. Wiles M, Duffy A, Neill K. The numerical translation of verbal probability expressions by patients and clinicians in the context of peri-operative risk communication. Anaesthesia.; 2020. p. e39-e45.

59. Gurmankin $\mathrm{AD}$, Baron $\mathrm{J}$, Armstrong $\mathrm{K}$. The effect of numerical statements of risk on trust and comfort with hypothetical physician risk communication. Medical Decision Making. 2004;24(3):265-271.

60. Zikmund-Fisher B, Smith D, Ubel P, Fagerlin A. Validation of the subjective numeracy scale (SNS): effects of low numeracy on comprehension of risk communications and utility elicitations. Medical Decision Making. 2007;27 https://doi.org/10.1177/0272989x07303824

Publisher's Note: Springer Nature remains neutral with regard to jurisdictional claims in published maps and institutional affiliations. 